\title{
Very Narrow Resonances in Spherical Proton Emitting Nuclei
}

\author{
T.N. Leite and N. Teruya \\ Departamento de Física, Universidade Federal da Paraíba and \\ C.P. 5008, 58051-970 João Pessoa, PB, Brazil. \\ H. Dias \\ Nuclear Theory and Elementary Particle Phenomenology Group, \\ Instituto de Física, Universidade de São Paulo and \\ C.P. 66318, 05315-970 São Paulo, SP, Brazil.
}

\begin{abstract}
The separation energy and half-life of some heavy proton emitting nuclei, and the single-particle structure of the unbound ${ }^{11} N$, have been evaluated by implementing a careful numerical treatment to solve Schrödinger equation in a continuum discretization context. The basic scheme behind the method consists in using the ground-state proton emitter in connection with an isolated single-particle resonance.
\end{abstract}

Keywords: Decay by proton emission; lifetimes; single-particle levels.

Journal-ref: International Journal of Modern Physics E, Vol. 11, No. 6 (2002) 469-473.

Recently, several unstable proton emitting nuclei with medium and heavy masses [1, 2, 3, 4, 5, 6, 7, 8, 9, 10, 11] have been discovered and are attracting much attention in both theoretical and experimental nuclear physics. In the light mass limit unbound ${ }^{11} N$ is another well studied proton emitter nucleus which presents some intriguing phenomena such as the $s_{1 / 2}$ intruder level and the ${ }^{11} \mathrm{Be}$ mirror states discussed in the recent literature $\left.12,13,14\right]$. The parent nucleus decays by proton emission in a quantum tunneling process. In a first approximation we can treat this problem as an unbound proton + core system in which the ground-states instabilities are studied from the single-particle resonance point of view. These resonances have large half-lives as long as $\sim 1 n s-1 s$. One of the great difficulties presented in these calculations is the determination of the separation energies and half-lives because these states have a long lifetime in comparison with the oscillation time inside the potential well: the energies are of the order of $\sim 1 \mathrm{MeV}$ and are extremely narrow resonances $(\Gamma \lesssim$ $10^{-12} \mathrm{MeV}$ ) corresponding to the half-lives $t_{1 / 2} \gtrsim 1 \mathrm{~ns}$ (an enormous numerical computation time would be necessary to sweep some $M e V s$ of energy by steps of the order of their width 
size in search of the resonance position ). The spin-parity assignment to the measured levels is another arduous task, and because the proton decay has a strong dependence on the angular momentum, the spin-parity predictions for some ground-state proton emitters are assigned by matching the experimental half-life with the theoretical calculations such as the WKB method [5, 8]. Several theoretical methods [1, 6, 9, 10] have already been presented to calculate the half-lives and spins of these nuclei and we are presenting another method based on solving the Schrödinger equation in a continuum discretization context 15, 16]. The purpose of this work is to calculate the decay properties, such as energies and half-lives, of some proton emitters implementing a careful numerical treatment to this continuum projection method.

The ground-state proton emitters are treated in connection with an isolated long lifetime single-particle resonance. Using orthogonal and complementary projectors the scattering solution $\left|\psi^{+}\right\rangle$of the single-particle Hamiltonian $H$ at energy $E$ can be written as [15]

$$
\left|\psi^{+}\right\rangle=|u\rangle\left\langle u \mid \psi^{+}\right\rangle+P\left|\psi^{+}\right\rangle,
$$

where $|u\rangle$ is a normalized single-particle state and $P=1-|u\rangle\langle u|$ projects onto the complementary sub-space of the single-particle Hilbert space. Projection and formal manipulation of the Schrödinger equation $(E-H)\left|\psi^{+}\right\rangle=0$ then gives

$$
\begin{gathered}
P\left|\psi^{+}\right\rangle=\left|\varphi^{+}\right\rangle+G_{P P}^{+}(E) P H|u\rangle\left\langle u \mid \psi^{+}\right\rangle, \\
\left\langle u \mid \psi^{+}\right\rangle=\frac{\left\langle u|H| \varphi^{+}\right\rangle}{E-\langle u|H| u\rangle-\left\langle u\left|H G_{P P}^{+}(E) H\right| u\right\rangle},
\end{gathered}
$$

where $G_{p p}^{+}(E)=\left(E+i \eta-H_{p p}\right)^{-1}$ is the Green's function and $\left|\varphi^{+}\right\rangle$is the scattering solution of the projected Hamiltonian $H_{p p}$ at energy $E$. The $\left|\varphi^{+}\right\rangle$solution is obtained by solving the inhomogeneous equation

$$
(E-H)\left|\varphi^{+}\right\rangle=\alpha|u\rangle,
$$

with $\alpha=-\left\langle u|H| \varphi^{+}\right\rangle$adjusted to satisfy the orthogonal relation $\left\langle u \mid \varphi^{+}\right\rangle=0$. The resonant energy, $E_{0}$, is defined as the energy for which the integrated internal probability density is largest and $|u\rangle$ is chosen as being proportional to the internal part of the resonant wave func- 
tion $\left|\psi^{+}\right\rangle_{E=E_{0}}$, truncated at a radius somewhat larger than the potential radius, containing the principal behaviour of the resonance inside the well:

$$
\langle r \mid u\rangle=N\left(1+e^{\left(r-R_{u}\right) / a_{u}}\right)^{-1}\left\langle r \mid \psi^{+}\right\rangle_{E=E_{0}},
$$

where $N$ is a normalization factor, $R_{u}$ and $a_{u}$ are the truncation parameters for $\langle r \mid u\rangle$. Thus the complex single-particle resonance energy $\left(\varepsilon_{u}-\frac{i \Gamma_{u}}{2}=\langle u|H| u\rangle+\left\langle u\left|H G_{P P}^{+}(E) H\right| u\right\rangle\right)$ is calculated in the energy $E_{0}$. The imaginary part $\left(\Gamma_{u}=2 \pi|\alpha|^{2}\right)$ corresponds to the singleparticle width and the associated half-life is given as $t_{1 / 2}=\frac{\hbar}{\Gamma_{u}} \ln 2$. The potential utilized in the single-particle Hamiltonian contains the central potential in a Woods-Saxon form and the spin-orbit term with a Woods-Saxon derivative form, also including the centrifugal and Coulomb (as a uniformly charged sphere) terms.

To determine the resonance parameters for the ground-state proton emitters, special attention must be taken in the numerical implementation of this method because these very narrow resonances are difficult to detect and require a more accurate search. The singleparticle resonance energy $\varepsilon_{u}$ is obtained imposing that it must reproduce the experimental $Q$ value by adjusting the central and spin-orbit potentials. To avoid wasting much computational time, and to achieve a better accuracy in the results, we start with an initial energy range near the experimental $Q$ value to solve the Schrödinger equation. The energy range is swept with a integration energy step $\Delta E$ to localize the energy $E_{0}$ where $\varepsilon_{u}$ and $\Gamma_{u}$ are calculated. After this, a new energy range is defined near $\varepsilon_{u}$. The range size is fixed at $\sim 2 \times 10^{3} \Delta E$ centered in $\varepsilon_{u}$, and it is swept again with a energy improvement in the integration step $\Delta E$, making a new $E_{0}$ determination in which the $\varepsilon_{u}$ and $\Gamma_{u}$ are recalculated. This recurrent relation is followed until the $\varepsilon_{u}$ and $\Gamma_{u}$ values became stable. In this way we search these resonances, sweeping successive energy ranges (sized about $2 \times 10^{3} \Delta E$ centered in $\varepsilon_{u}$ ), by improving the integration energy step. The Woods-Saxon central and spin-orbit

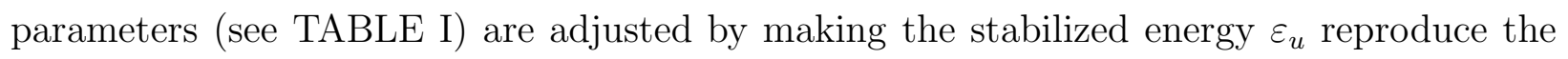

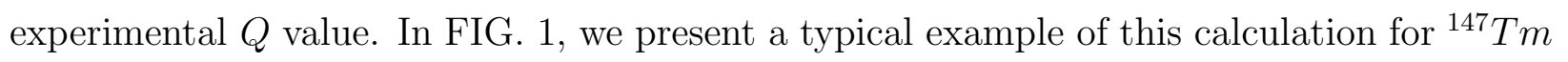
for which the potential was adjusted to reproduce the experimental ground-state energy. In TABLE I our results are presented in comparison with the other methods showing a good agreement between the data. The small differences between our calculations and the experimental results are due to the fact that we assume a pure single-particle configuration in a spherical model while the real system may have soft deformations or mixing configurations 
TABLE I: The Woods-Saxon central and spin-orbit parameters used in the calculations.

\begin{tabular}{|c|c|c|c|}
\hline \multicolumn{4}{|c|}{$\begin{array}{l}V_{R}(r)=V_{0 R} f(r) ; V_{l s}(r)=V_{0 l s}\left(\frac{\hbar}{m_{\pi} c}\right)^{2} \frac{1}{r} f^{\prime}(r) \vec{l} \cdot \vec{s} \\
f(r)=\frac{1}{1+e^{(r-R) / a}} ; R=1.17 A^{1 / 3} ; a=0.75 f m ; V_{0 l s}=6.01 M e V\end{array}$} \\
\hline \multicolumn{2}{|c|}{ Nuclide $V_{0 R}(M e V)$} & \multicolumn{2}{|c|}{ Nuclide $V_{0 R}(M e V)$} \\
\hline${ }^{109} I$ & 61.699 & ${ }^{155} \mathrm{Ta}$ & 61.955 \\
\hline${ }^{145} \mathrm{Tm}$ & 63.079 & ${ }^{167} \mathrm{Ir}$ & 59.700 \\
\hline${ }^{147} \mathrm{Tm}$ & 60.398 & ${ }^{185} \mathrm{Bi}$ & 57.875 \\
\hline${ }^{151} \mathrm{Lu}$ & 63.000 & & \\
\hline
\end{tabular}

TABLE II: The half-lives calculated in this work are compared with the other proton emitters results. The Woods-Saxon and spin-orbit parameters are adjusted to fit the experimental energies.

\begin{tabular}{ccccccc}
\hline \hline & \multicolumn{5}{c}{ This work Ref.[1] Ref.[10] } & Exp. \\
\hline Nuclide & Orbit $Q(\mathrm{MeV})$ & $T_{1 / 2}$ & $T_{1 / 2}$ & $T_{1 / 2}$ & $T_{1 / 2}$ \\
\hline${ }^{109} \mathrm{I}$ & $2 d_{5 / 2}$ & 0.829 & $7.5 \mu \mathrm{s}$ & $10 \mu \mathrm{s}$ & - & $(100 \pm 5) \mu s[2]$ \\
${ }^{145} \mathrm{Tm}$ & $1 h_{11 / 2}$ & 1.728 & $1.5 \mu s$ & - & - & $(3.5 \pm 0.1) \mu s[5]$ \\
${ }^{147} \mathrm{Tm}$ & $2 d_{3 / 2}$ & 1.132 & $280.0 \mu \mathrm{s}$ & $210 \mu s$ & $206.8 \mu s$ & $(360 \pm 40) \mu s[2]$ \\
${ }^{151} \mathrm{Lu}$ & $1 h_{11 / 2}$ & 1.255 & $165.2 m s$ & $60 m s$ & $58.4 m s$ & $\left(130_{-50}^{+160}\right) \mathrm{ms}[2]$ \\
${ }^{155} \mathrm{Ta}$ & $1 h_{11 / 2}$ & 1.776 & $8.6 \mu s$ & - & - & $\left(12_{-3}^{+4}\right) \mu s[8]$ \\
${ }^{167} \mathrm{Ir}$ & $3 s_{1 / 2}$ & 1.086 & $193.3 \mathrm{~ms}$ & $36 m s$ & - & $(110 \pm 15) \mathrm{ms}[4]$ \\
${ }^{185} \mathrm{Bi}$ & $3 s_{1 / 2}$ & 1.611 & $3.6 \mu s$ & $3.2 \mu s$ & $3.17 \mu s$ & $(44 \pm 16) \mu s[3]$ \\
\hline \hline
\end{tabular}

altering the occupation probabilities.

In the light proton unbound nucleus ${ }^{11} N$, the instability requires shorter decay times than those in the heaviest ones. In this nucleus the ground-state and the first excited state have spin-parity inversion as in its mirror ${ }^{11} \mathrm{Be}$. Recent experimental data [13, 14] report the energies and widths of the ground and excited states in ${ }^{11} N$. In Ref. [13] the measured resonances are identified by adjusting the potential to reproduce the best fit to the data. Using the same Woods-Saxon central and spin-orbit parameters as those given in Ref. [13] we calculate the ground-state intruder $\frac{1}{2}^{+}$and the other excited states. The results are showed in TABLE III in comparison with the data of Refs. 13] and [14]. We can see that the resonant energies present good agreement with the experimental data while the widths 


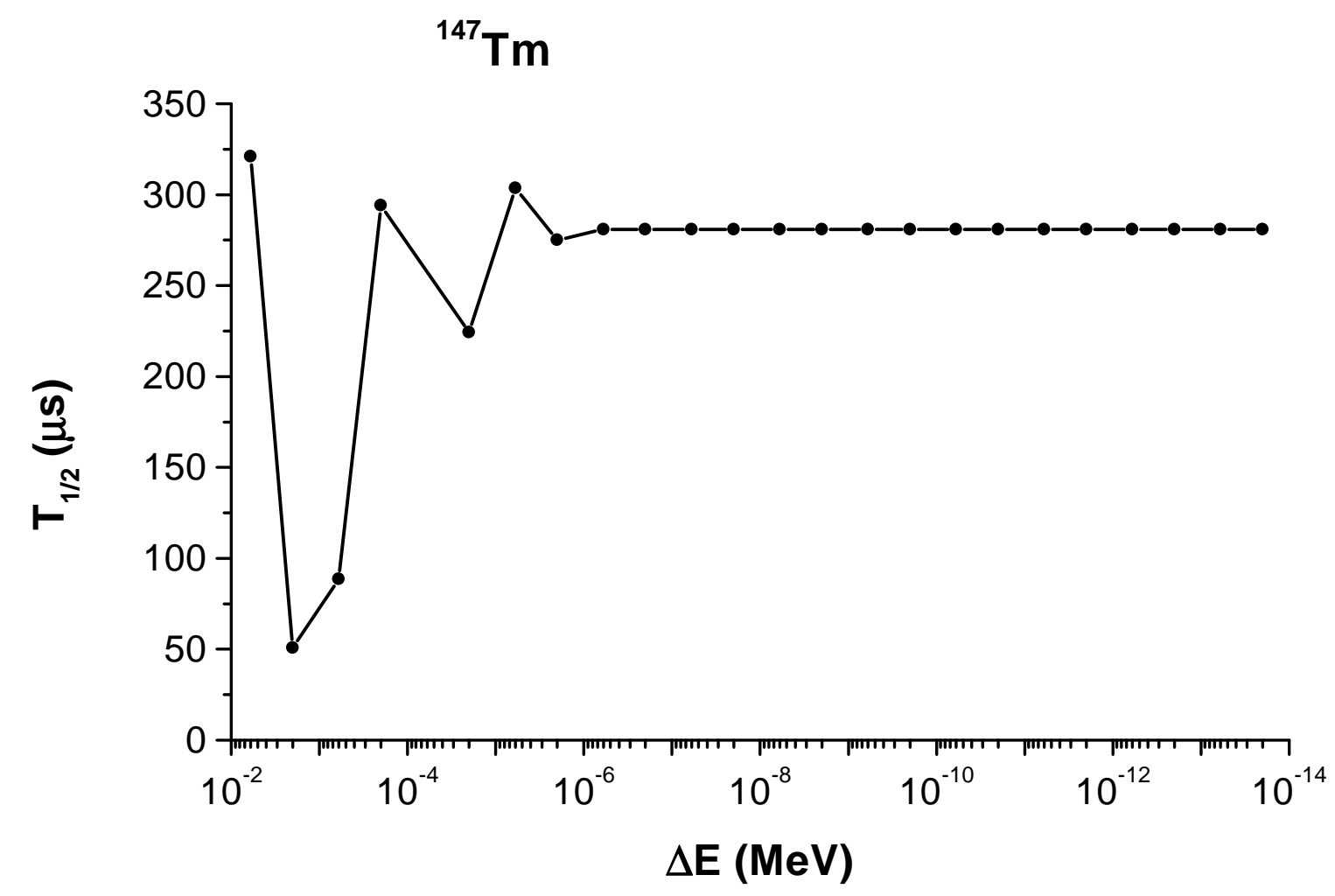

FIG. 1: The half-life for ${ }^{147} \mathrm{Tm}$ is calculated by improving the integration energy step, $\Delta E$, that means a refinement in the energy range where the resonance is evaluated. The results became stable when the energy precision is improved.

have small discrepancies. The major variation in the results is due to the $\frac{1}{2}^{+}$ground-state level which is a broad low energy resonance. In this case, the localization of the resonance shows up as the opposite problem to the one of the heaviest proton emitters, because here, the resonance width is as broad as the value of its position in energy, indicating that the half-life is approximately identical to its short oscillation time inside the well, which brings some uncertainty in the evaluation of the decay properties.

In short, the half-lives of some proton emitters are evaluated by implementing a careful numerical treatment to solve Schrödinger equation in a continuum discretization approach. The results are in good agreement with the other theoretical and experimental data, showing that this method offers an efficient alternative for predicting the ground and excited states in proton emitting nuclei and these are necessary elements in more complex nuclear structure 
TABLE III: The calculated single-particle resonances for unbound ${ }^{11} N$ are presented in comparison with the experimental data.

\begin{tabular}{|c|c|c|c|c|c|c|}
\hline \multirow{2}{*}{$\begin{array}{l}{ }^{11} N \\
I^{\pi}\end{array}$} & \multicolumn{2}{|c|}{ This work } & \multicolumn{2}{|c|}{ Ref.[13] } & \multicolumn{2}{|c|}{ Ref.[14] } \\
\hline & $E(M e V)$ & $\Gamma(M e V)$ & $E(M e V)$ & $\Gamma(M e V)$ & $E(M e V)$ & $\Gamma(M e V)$ \\
\hline$\frac{1}{2}^{+}$ & 1.38 & 0.59 & 1.27 & 1.44 & $1.63(5)$ & $0.4(1)$ \\
\hline$\frac{1}{2}^{-}$ & 2.18 & 0.51 & 2.01 & 0.84 & $2.16(5)$ & $0.25(8)$ \\
\hline$\frac{5}{2}^{+}$ & 3.77 & 0.47 & 3.75 & 0.60 & $3.61(5)$ & $0.50(8)$ \\
\hline$\frac{3}{2}^{+}$ & 4.81 & 0.86 & 4.50 & 1.27 & - & - \\
\hline
\end{tabular}

calculations for evaluating structures other than the single-particles ones.

\section{Acknowledgments}

This work was supported in part by Conselho Nacional de Desenvolvimento Científico e Tecnológico (CNPq), Brazil.

[1] S. Aberg, P. B. Semmes and W. Nazarewicz, Phys. Rev. C 56, 1762 (1997).

[2] P.J. Sellin et al., Phys. Rev. C 47, 193 (1993).

[3] C.N. Davids et al., Phys. Rev. Lett. 76, 592 (1996).

[4] C.N. Davids et al., Phys. Rev. C 55, 2255 (1997).

[5] J.C. Batchelder et al., Phys. Rev. C 57, R1042 (1998).

[6] E. Maglione, L.S. Ferreira and R.J. Liotta, Phys. Rev. Lett. 81, 538 (1998).

[7] C.H. Yu et al., Phys. Rev. C 59, R1834 (1999).

[8] J. Uusitalo et al., Phys. Rev. C 59, R2975 (1999).

[9] C.N. Davids and H. Esbensen, Phys. Rev. C 61, 054302 (2000).

[10] P. Talou, N. Carjan, C. Negrevergne and D. Strottman, Phys. Rev. C 62, 014609 (2000).

[11] K. Starosta et al., Phys. Rev. C 62, 044309 (2000).

[12] S. Aoyama, Phys. Rev. C 62, 034305 (2000).

[13] K. Markenroth et al., Phys. Rev. C 62, 034308 (2000).

[14] J.M. Oliveira, Jr. et al., Phys. Rev. Lett. 84, 4056 (2000). 
[15] N. Teruya, A.F.R. de Toledo Piza and H. Dias, Phys. Rev. C 44, 537 (1991).

[16] N.Teruya, H.Dias and A.F.R. de Toledo Piza, Nuc. Phys. A 556, 157 (1993). 